FOUNDATIONS

ADVANCES

\title{
To superspace and beyond
}

\author{
Mark S. Senn*
}

Department of Chemistry, Inorganic Chemistry Laboratory, University of Oxford, South Parks Road, Oxford OX1 3QR, United Kingdom, and Department of Chemistry, University of Warwick, Gibbet Hill, Coventry CV4 7AL, United Kingdom. *Correspondence e-mail: m.senn@warwick.ac.uk

Keywords: structure-property relationships; superspace; incommensurate structures; irreducible representations; charge-density waves; phase transitions.

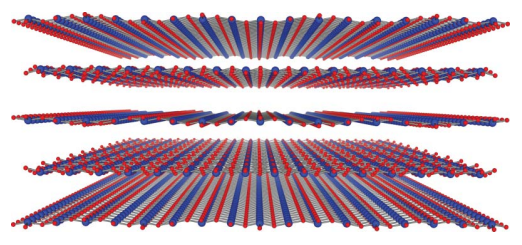

C 2017 International Union of Crystallography
Much progress has been made in understanding the structure-property relationship over the past few decades. This is partly due to our improved ability to measure the subtle structural distortions that are either linked to the development of a specific physical property or that lead to its suppression. A very significant contribution in this field has also been the development of the language required to formally describe such symmetrybreaking events that characterize the order parameters that drive these phase transitions. However, until now, the tools available have only allowed for the exploration of the symmetry implications of order parameters corresponding to commensurate modulations. In this issue, Stokes \& Campbell (2017) present an algorithm for computing the allowed subgroups arising from any number of incommensurate order parameters and for identifying the resulting $(3+d)$-dimensional superspace groups. They also describe a practical implementation of this algorithm for cases consisting of up to three arbitrary independent modulations. This work provides key tools for understanding the structureproperty relationships of the many technologically important materials that display incommensurate modulations in their atomic and/or magnetic structure.

While Landau theory has been widely used since the 1960s to describe second-order phase transitions (Cowley, 1980), often the starting point has been to use an order parameter that is phenomenological. That is to say, the order parameter is related to an observation of the physical property, such as the magnitude of the measured electrical or magnetic polarization. However, this need not be the case, and indeed formally each order parameter that contributes to the phase transition (leading to a child structure) must transform as an irreducible representation (Campbell et al., 2006) of the highsymmetry (parent) structure. Or put another way, it must be an allowed excitation of the parent structure, which in the harmonic approximation will transform as an irreducible representation of the parent space group. With the use of invariant analysis (Hatch \& Stokes, 2003), this allows a full expansion of the free energy to be performed about the parent structure in terms of the the allowed excitation(s) beyond just the harmonic (second) order, provided that the secondary 'symmetry-allowed' couplings of the primary order parameter can be elucidated.

As crystallographers, experimentally what we determine is the positions of the atoms above and below a phase transition. If we are to pursue the analysis described above, then we must identify the set of atomic displacements that lead to a phase transition from parent to child structure. One may then decompose these symmetry-breaking distortions into order parameters of the irreducible representations of the parent space group. This process may simply be thought of as a change of basis to one which is orthogonal in the symmetry space of the parent structure. A substantial bottleneck to all of this analysis is hence not just our ability to measure subtle structural distortions, but our ability to classify these subtle orderings in terms of irreducible representations. Although the first complete tabulation of (time-even) irreducible representations (for special points in k-space) was made in the 1970s (Bradley \& Cracknell, 1972), the analysis of structural distortions in terms of these remained the preserve of experts in the field of group theory. The development of the online tool ISODISTORT by Campbell, Stokes, Tanner and Hatch in 2006 (Campbell et al., 2006) finally bridged this gap, making this analysis trivial even for structures undergoing the most complicated phase transitions driven by order parameters that consist of multiple superposed irreducible representations. 


\section{scientific commentaries}
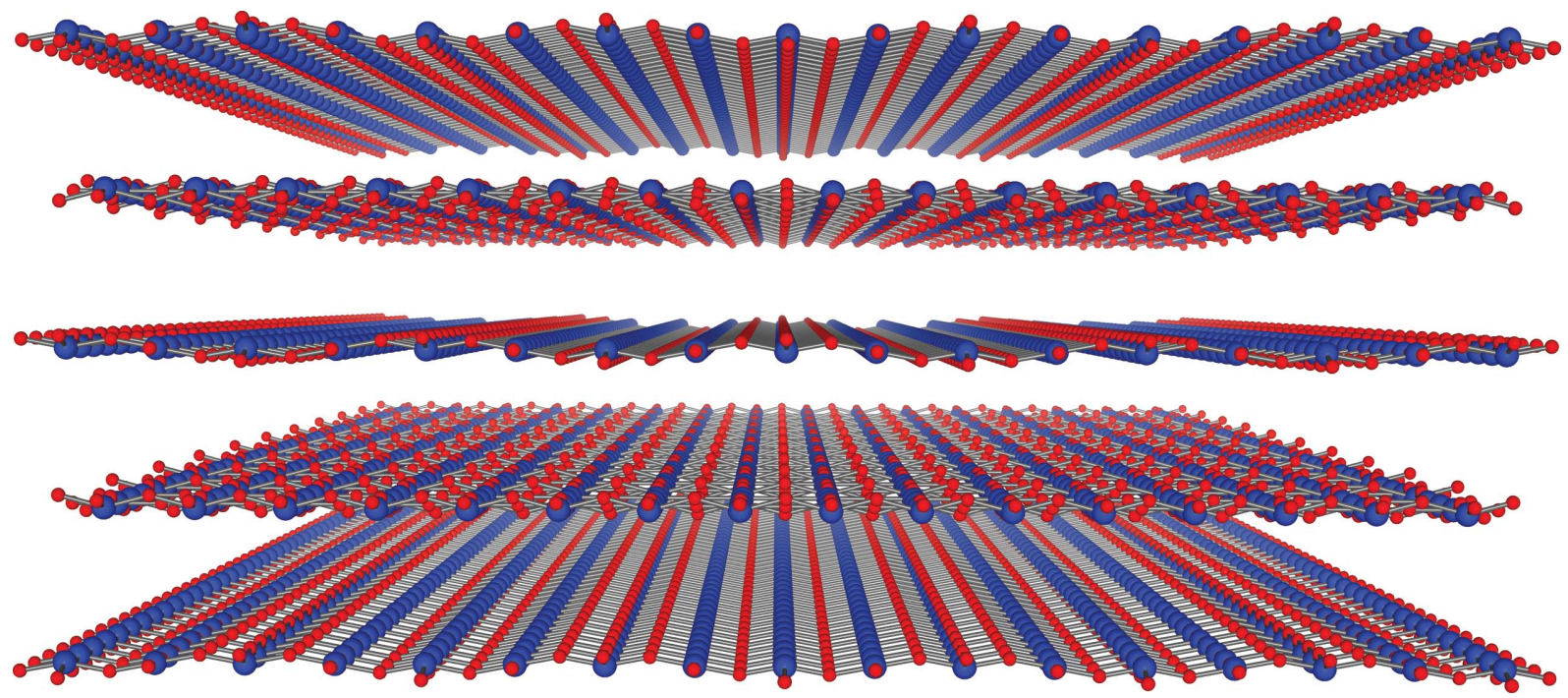

Figure 1

An exaggerated illustration of a possible incommensurate modulation of $\mathrm{CuO}_{4}$ planes $(\mathrm{Cu}$ in blue, $\mathrm{O}$ in red) such as found in the layered cuprate superconductors $\mathrm{La}_{2-x} \mathrm{Sr}_{x} \mathrm{CuO}_{4}$ with parent $14 / \mathrm{mmm}$ symmetry. Although weak superstructure peaks have been measured that are associated with a charge-density wave with a propagation vector $\mathbf{k}=\left[0.23,0, \frac{1}{2}\right]$ (Croft et al., 2014), the limited number of weak observed reflections, the large number of possible isotropy subgroups and the number of degrees of freedom within each of these models mean that the symmetry of the CDW phase in this and other related systems is still in question. The figure is drawn using ISODISTORT (Campbell et al., 2006) and VESTA (Momma \& Izumi, 2011).

The success of this analysis is evident in the literature. A large number of complex phase transitions have been interpreted thanks to these tools, giving physical insight into processes such as charge ordering, ferroelectricity, magnetic exchange anisotropy and negative thermal expansion (Senn $e t$ al., 2012; Kocsis et al., 2014; Khalyavin, Salak, Lopes et al., 2015; Orlandi et al., 2016; Khalyavin, Salak, Manuel et al., 2015; Senn et al., 2015). Other works have devised approaches based on this analysis to effectively search and determine space groups of materials that have undergone complex phase transitions (Kerman et al., 2012; Lewis et al., 2016). Rietveld and single-crystal refinement programs such as Topas and FullProf can now refine models directly in this symmetryadapted basis, and more recently this approach has even been applied to directly fitting pair distribution function data (Senn et al., 2016).

Crucially, it is only the assignment of the full (isotropy) subgroup symmetry that allows identification of all of the secondary order parameters. This is vital not only for correctly constructing the Landau-style free energy expansion as mentioned above, but also for identifying and predicting coupling mechanisms within the solid state that can give rise to unexpected physical properties. The recent resurgence of interest in improper ferroelectrics, where the polarization forms only a secondary order parameter of two antiferrodistortive primary order parameters, is a good example of this, and undoubtedly this has been driven to a great part by the ease with which such couplings can now be ascertained [see Benedek et al. (2015) and Young et al. (2015) for some recent review articles].

However, there remains a final frontier. Many crystal structures display incommensurate phase transitions that are related to unusual lattice dynamics and charge modulations where phonons go soft at general points in reciprocal space, rather than at commensurate wavevectors. In the article A general algorithm for generating isotropy subgroups in superspace by Stokes and Campbell, not only is the first algorithm presented for enumerating all of the possible subgroups associated with the action of an order parameter with incommensurate propagation vectors, but the machinery is also provided so that it may be undertaken by a non-expert in the field. Undoubtedly this work will inspire renewed efforts to tackle problems associated with incommensurate crystallography. Where solutions exist, they may be decomposed in terms of irreducible representations of the parent space groups, and new physical insights gained into secondary couplings. Where structural models have yet to be found, possible subgroups can be enumerated thanks to these new online tools (Stokes et al., 2016) and tested systematically against diffraction data (Lewis et al., 2016).

The importance of using the full magnetic symmetry group to describe incommensurate magnetic structures has been highlighted recently (Perez-Mato et al., 2012). Here magnetic superspace groups not only provide a complete description of the ordering with a minimum number of refinable parameters, but they also serve to reveal any secondary magneto-structural couplings such as are vital for understanding type-II multiferroic mechanisms. But maybe the most significant challenge to be undertaken in this field is in modelling the incommensurate charge-density wave (CDW) phases found in many layered cuprates, which is now generally accepted as being associated with a suppression of the superconducting state (Wu et al., 2011; Ghiringhelli et al., 2012) (see Fig. 1 for further discussion). Ultimately a full symmetry analysis of these systems in their CDW state should reveal the spinlattice coupling interactions that are responsible for mediating superconductivity. Equipped with these new tools for exploring the consequences of symmetry-lowering phase 


\section{scientific commentaries}

transitions, we can now tackle such problems and bravely go 'to superspace and beyond'.

\section{References}

Benedek, N. A., Rondinelli, J. M., Djani, H., Ghosez, P. \& Lightfoot, P. (2015). Dalton Trans. 44, 10543-10558.

Bradley, C. J. \& Cracknell, A. P. (1972). The Mathematical Theory of Symmetry in Solids: Representation Theory for Point Groups and Space Groups. Oxford: Clarendon Press.

Campbell, B. J., Stokes, H. T., Tanner, D. E. \& Hatch, D. M. (2006). J. Appl. Cryst. 39, 607-614.

Cowley, R. A. (1980). Adv. Phys. 29, 1-110.

Croft, T. P., Lester, C., Senn, M. S., Bombardi, A. \& Hayden, S. M. (2014). Phys. Rev. B, 89, 224513.

Ghiringhelli, G. et al. (2012). Science, 337, 821-825.

Hatch, D. M. \& Stokes, H. T. (2003). J. Appl. Cryst. 36, 951-952.

Kerman, S., Campbell, B. J., Satyavarapu, K. K., Stokes, H. T., Perselli, F. \& Evans, J. S. O. (2012). Acta Cryst. A68, 222-234.

Khalyavin, D. D., Salak, A. N., Lopes, A. B., Olekhnovich, N. M., Pushkarev, A. V., Radyush, Y. V., Fertman, E. L., Desnenko, V. A., Fedorchenko, A. V., Manuel, P., Feher, A., Vieira, J. M. \& Ferreira, M. G. S. (2015). Phys. Rev. B, 92, 224428.

Khalyavin, D. D., Salak, A. N., Manuel, P., Olekhnovich, N. M., Pushkarev, A. V., Radysh, Y. V., Fedorchenko, A. V., Fertman,
E. L., Desnenko, V. A. \& Ferreira, M. G. S. (2015). Z. Kristallogr. 230, 767-774.

Kocsis, B., Perez-Mato, J. M., Tasci, E. S., de la Flor, G. \& Aroyo, M. I. (2014). J. Appl. Cryst. 47, 1165-1179.

Lewis, J. W., Payne, J. L., Evans, I. R., Stokes, H. T., Campbell, B. J. \& Evans, J. S. O. (2016). J. Am. Chem. Soc. 138, 8031-8042.

Momma, K. \& Izumi, F. (2011). J. Appl. Cryst. 44, 1272-1276.

Orlandi, F., Righi, L., Mezzadri, F., Manuel, P., Khalyavin, D. D., Delmonte, D., Pernechele, C., Cabassi, R., Bolzoni, F., Solzi, M. \& Calestani, G. (2016). Inorg. Chem. 55, 4381-4390.

Perez-Mato, J. M., Ribeiro, J. L., Petricek, V. \& Aroyo, M. I. (2012). J. Phys. Condens. Matter, 24, 163201.

Senn, M. S., Bombardi, A., Murray, C. A., Vecchini, C., Scherillo, A., Luo, X. \& Cheong, S. W. (2015). Phys. Rev. Lett. 114, 035701.

Senn, M. S., Keen, D. A., Lucas, T. C. A., Hriljac, J. A. \& Goodwin, A. L. (2016). Phys. Rev. Lett. 116, 207602.

Senn, M. S., Wright, J. P. \& Attfield, J. P. (2012). Nature, 481, 173176.

Stokes, H. T. \& Campbell, B. J. (2017). Acta Cryst. A73, 4-13.

Stokes, H. T., van Orden, S. \& Campbell, B. J. (2016). J. Appl. Cryst. 49, 1849-1853.

Wu, T., Mayaffre, H., Krämer, S., Horvatić, M., Berthier, C., Hardy, W. N., Liang, R., Bonn, D. \& Julien, M. (2011). Nature, 477, 191194.

Young, J., Stroppa, A., Picozzi, S. \& Rondinelli, J. M. (2015). J. Phys. Condens. Matter, 27, 283202. 